\title{
International Classification of Diseases for Oncology
}

National Cancer Institute

\section{Source}

National Cancer Institute. International Classification of Diseases for Oncology. NCI

Thesaurus. Code C37978.

The International Classification of Diseases for Oncology has been used for nearly 25 years as a tool for coding diagnoses of neoplasms in tumor and cancer registrars and in pathology laboratories. ICD-O is a dual classification with coding systems for both topography and morphology. The topography code describes the site of orig in of the neoplasm. The morphology code describes the characteristics of the tumor itself, including its cell type and biologic activity. (from Amazon.com) 